\title{
VÉGÉTATION DE LA MACARONÉSIE
}

La flore et la végétation de la Macaronésie (Iles du Cap-Vert, Canaries, Madère, Açores) ont été l'objet d'études plus ou moins approfondies depuis un siècle. La bibliographie sommaire ci-jointe permet de poser des jalons sur le cheminement de nos connaissances. On constatera que la flore a engagé les préoccupations des chercheurs d'une façon beaucoup plus intensive que la végétation.

Il va de soi que l'analyse de la couverture végétale, et éventuellement des associations ou communautés végétales, ne saurait se faire sans une connaissance détaillée de l'identité taxonomique des plantes. Une fois fait ce catalogue, le phytogéographe s'adonne à la cartographie des espèces et à la détection de leurs affinités historiques. Il peut donc reconnaître certains éléments floristiques et éventuellement assigner à chaque espèce une appartenance qui caractérise ses origines et son patron de distribution.

La flore macaronésienne comprend donc des éléments divers, témoins des vicissitudes géologiques et historiques qu'ont connu les archipels.

Un vieux stock tertiaire dont certains éléments sont connus à l'état fossile en Europe est particulièrement adapté à la forêt ombrophile tempérée, ou laurisilva (ex.: Laurus canariensis, Apollonias barbujana).

Les espèces d'affinité méditerranéenne sont assez nombreuses et dominent souvent les maquis ou fourrés (ex.: Cistus symphytifolius, Smilax canariensis). 

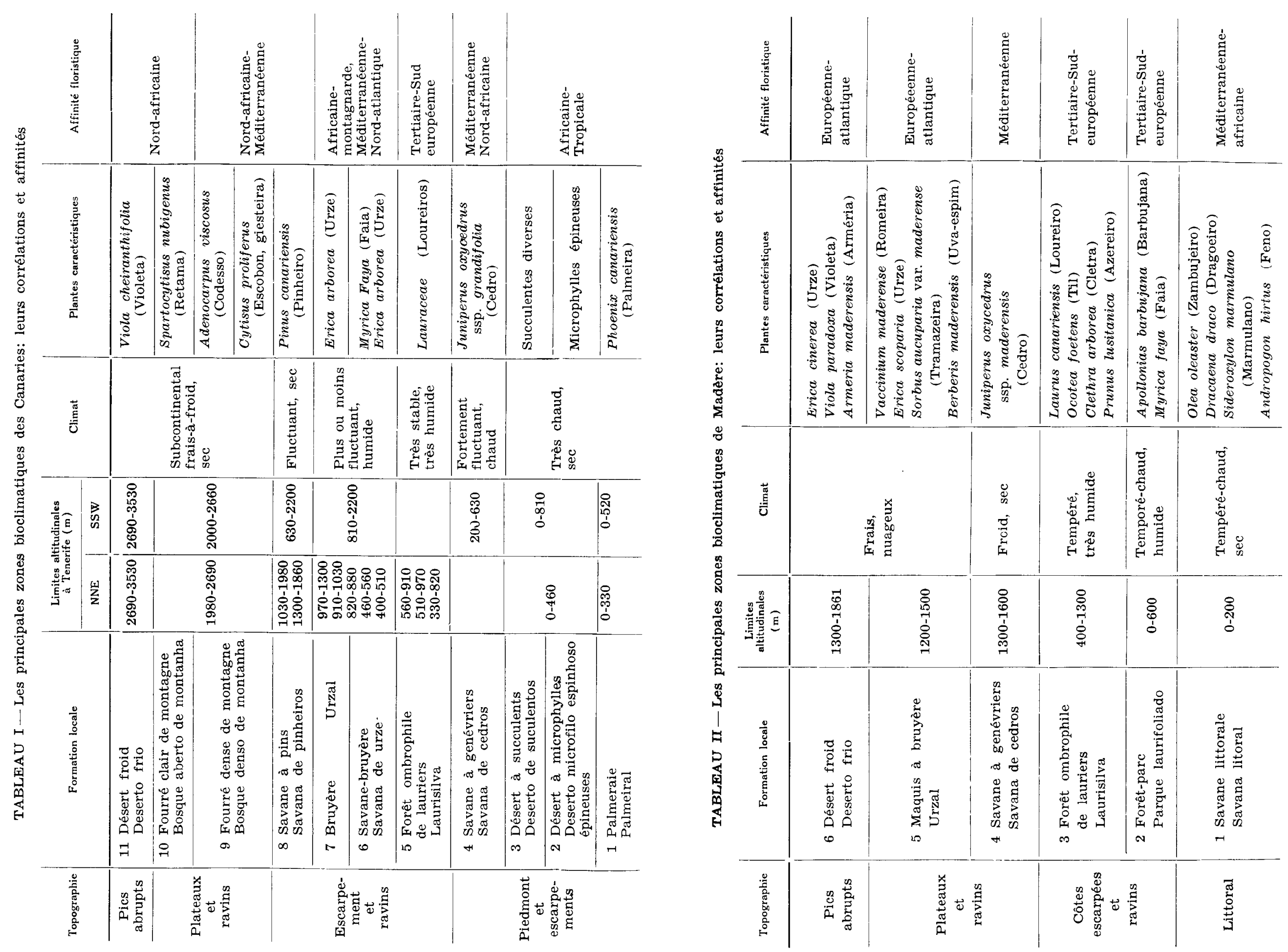


\begin{tabular}{|c|c|c|c|c|c|c|}
\hline 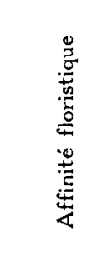 & 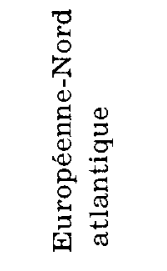 & 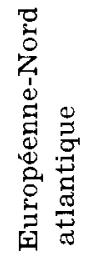 & 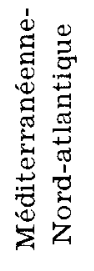 & 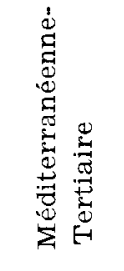 & 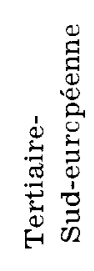 & 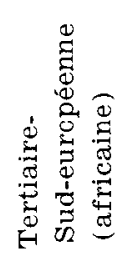 \\
\hline 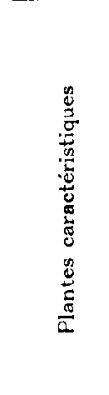 & 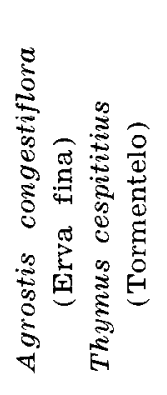 & 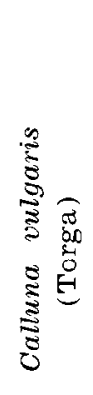 & 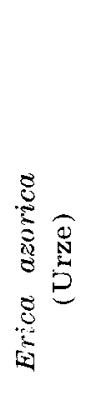 & 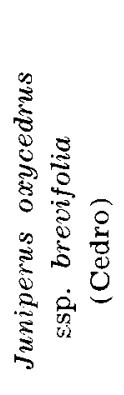 & 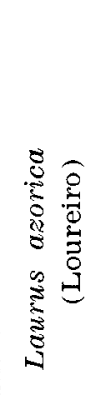 & 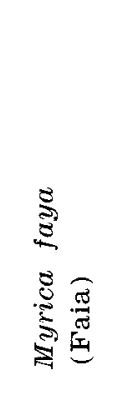 \\
\hline 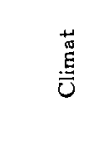 & \multicolumn{2}{|c|}{ 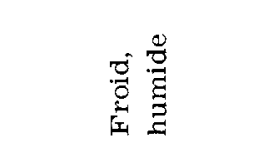 } & \multicolumn{3}{|c|}{ 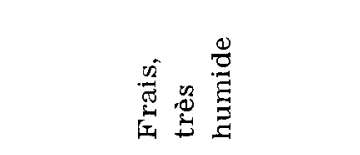 } & 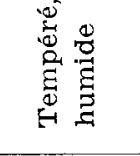 \\
\hline 总 & 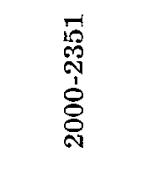 & 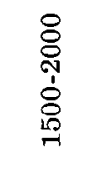 & $\begin{array}{l}8 \\
\stackrel{0}{0} \\
\stackrel{1}{1} \\
\stackrel{8}{8}\end{array}$ & $\begin{array}{l}8 \\
\text { के } \\
\text { षे. }\end{array}$ & 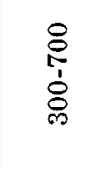 & $\begin{array}{l}\stackrel{8}{0} \\
\vdots \\
0\end{array}$ \\
\hline 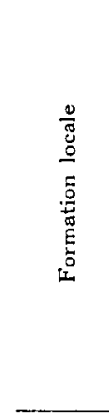 & 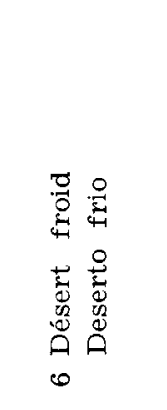 & 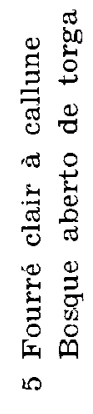 & 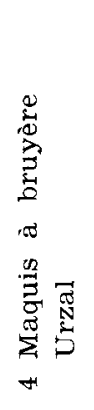 & 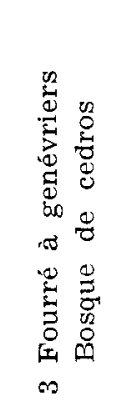 & 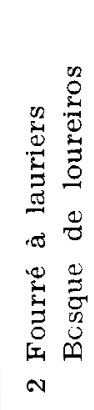 & 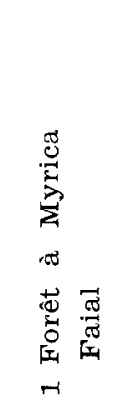 \\
\hline 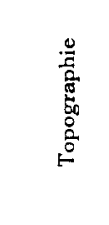 & \multicolumn{2}{|c|}{ 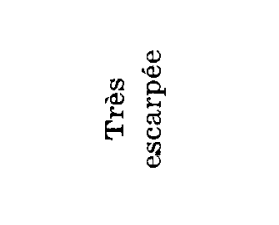 } & \multicolumn{3}{|c|}{ 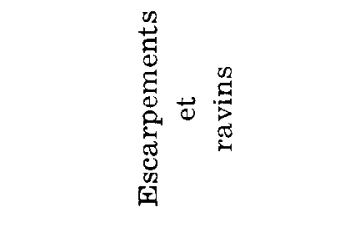 } & 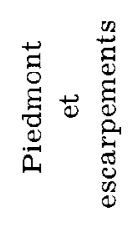 \\
\hline
\end{tabular}

Des plantes caractéristiques du milieu forestier humide boréo-atlantique sont fréquentes dans les forêts (ex.: Dryopteris filixmas, Erica scoparia).

Les déserts africains ont contribué beaucoup au peuplement littoral chaud et sec (ex.: Euphorbia canariensis, Aeonium tabuiare).

La zone sèche sud-africaine est également à l'origine de certaines espèces (ex.: Kleinia neriifolia).

Les montagnes africaines, d'autre part, sont aussi à l'origine de quelques espèces (ex.: Erica arborea, Hypericum grandifolium).

Il reste un stock de plantes plus ou moins cosmopolites (ex.: Pteridium aquilinum) et un grand nombre d'espèces naturalisées (ex.: Opuntia ficus-indica, Eupatorium riparium).

Encore que la distribution des espèces dans les diverses zones de végétation soit plus ou moins prévisible, tel qu'indiqué plus haut, il reste à mesurer séparément la zone climatique et la zone de végétation.

A l'aide de climogrammes on établit facilement la prédominance de régimes différents: océanique, méditerranéen montagnard et désertique. Ce dernier est nettement influent sur le littoral canarien. Le régime méditerranéen, d'autro part, est sensible sur le littoral madérien et fort atténué ailleurs. Le régime océanique (modéré, très humide) est le plus fort aux Açores, alors qu'il est bien développé à Madère, mais localisé aux Canaries. Le régime montagnard peu sensible à Madère est dominant aux plus hautes altitudes aux Canaries et aux Açores.

Les trois tableaux ci-joints donnent une idée de la distribution des zones de végétation dans ces trois archipels.

PIERRE DANSEREAU

\section{BIBLIOGRAPHIE SOMMAIRE}

Banneruan, David A. (1922) - The Canary Islands, their history, natural history and scenery. Gurney \& Jackson, London, $\mathrm{xv}+365 \mathrm{pp}$.

CEballos, L. \& ORTuNo, F. (1951) - Vegetación y flora florestal de las Canarias Occidentales. Inst. Forest. de Inv. y Exp., Madrid, 465 pp. 
Chevalier, A (1935) - «Les Iles du Cap Vert. Flore de l'Archipel». Rev. Appl. 15: 733-1090.

DansereaU, Pierre (1956) - «Les types de dispersion dans la forêt de laurier des Iles Canaries». Ann. de l'ACFAS 22: 60.

DANSEREAU, PIERRE (1957) - «A preliminary note on the structure variations of temperate rainforest》. Proc. 8th Pac. Sci. Congr., vol. IV (Botany): 407-436.

Dansereau, Pierre (1961a) - «Etudes macaronésiennes. I -- Géographie des Cryptogames vasculaires». Agronomia Lusitana 23(3): 151-181.

Dansereau, Pierre (1961b) - «Le rôle des genévriers dans la végétation des îles atlantiques». Ann. de l'ACFAS 27: 46.

Dansereat, Pierre (1965) — «Le contrôle de la végétation dans les îles océaniques». In: Simpósio: «Aportación de las Investigaciones Ecológicas y Agrícolas en la Lucha del Mundo contra el Hambre», Consejo Superior de Investigaciones Científicas, Madrid, ses. 5, 41 pp.

DANSEREAU PIERRE (1966) - «Etudes macaronésiennes. III - La zonation altitudinale». Nat. Canadien 93(6):779-795.

Dansereau Pierre (1968) - «Macaronesian studies. II - Structure and functions of the laurel forest in the Canaries». Collectanea Botanica 7(1:11)): 227-280.

DAVEAU, Jules (1889) - «Remarques sur la flore de l'Archipel des Açores». Soc. Carlos Ribeiro, Porto; Rev. Sciencias Naturaes e Sociaes, $\mathrm{n}^{\circ}$ 1, pp. 3-8.

Godman, F. DE C. (1870)-Natural history of the Azores. John Van Voorst, London, VII $+358 \mathrm{pp}$.

GuPPY, H. B. (1914) - «Notes on the native plants of the Azores as illustrated on the slopes of the Mountain of Pico». Roy. Bot. Gard., Kew, Bull. Misc. Inform., $\mathrm{n}^{\circ}$ 9, pp. 305-321.

LEMS, KoRnelius (1958) - Phytogeographic study of the Canary Islands. Thesis, Dept. Bot., Univ. Michigan, 2 volumes.

LeMS, KoRnelius (1960a) - «Floristic botany of the Canary Islands». Sarracenia $\mathrm{n}^{\circ} 5,94 \mathrm{pp}$.

LEMS, KoRNelius $(1960 \mathrm{~b})$ - «Botanical notes on the Canary Islands. II - The evolution of plant forms in the islands: Aeonium». Ecology 41(1): 1-17.

LEMS, KoRNeliUs (1961)- -Botanical notes on the Canary Islands. III - The life form spectrum and its interpretation». Ecology 42(3): $569-572$.

Lowe, A. TH. (1868)-A manual flora of Madeira and the adjacent islands of Porto Santo and the Desertas, vol. I - Dichlamydeae; vol. II, part I - Corolliflorae. London, XII +618 pp.; 113 pp.

MARLER, P. \& BOATMAN, D. J. (1952) - «An analysis of the vegetation of the northern slopes of Pico - the Azores》. Jour. Ecol. 40(1): 143-155.
MÉmoIRes DE LA SOCIÉTÉ DE BIOGÉOGRAPHIE, VIII (1946) - Contribution à l'étude du peuplement des îles atlantides. Paul Lechevalier, Paris, 500 pp.

Menezes, Carlos Azevedo de (1914) - Flora do Archipelago da Madeira. Junta Agricola da Madeira, Funchal, $282 \mathrm{pp}$

Palhinha, R. T. (1966) - Catálogo das plantas vasculares dos Açores. Soc. Est. Açorianos Afonso Chaves, Lisboa, $\mathrm{xv}+186 \mathrm{pp}$

PItARd, J. \& Proust, J. (1908) - Les Iles Canaries. Flore de l'archipel. Klincksieck, Paris, 503 pp. +10 pl.

Ribeiro, O. (1949) - 1. L'Ile de Madère. Maquis et forêts d'essences indigènes. 1:500000 (p. 38). 2. L'Ile de Madère. L'Utilisation du sol. 1:200 000. Carte V. In: L'Ile de Madère. Etude géographique. 16ème Congr. Int. Gécgr., Lisbonne.

RoUCH, M. J. (1946) - «Océanographie et climatologie des iles Atlantides». In: Contribution à l'étude du Peuplement des Iles Atlantides, Soc. Biogéogr. Paris, Mém. 8: 41-57.

Teles, A. N. (1965) - «Bibliographie des cartes de végétation du Portugal». Excerpta Botanica 6(4): 297-319.

TRELEASE, William (1897) - «Botanical observations on the Azores». Ann. Rep. Missouri Bot. Gard. 8: 77-220, pl. 12-66.

Tutin, T. G. (1953) -- «The vegetation of the Azores». Jour. Ecol. 41(1): 53-61.

WebB, PhILIP BARKER- \& BERTHELOT, SABIN (1836-1850) - Histoire naturelle des Iles Canaries, tome III, partie 2. Phytographia Canariensis. Béthune, Editeur, Paris, sect. 1, 229 pp.; sect. 2, 496 pp. sect. $3,479 \mathrm{pp}$. 